\title{
Generalized rate-equation analysis of excitation exchange between silicon nanoclusters and erbium ions
}

\author{
A. J. Kenyon, * M. Wojdak, and I. Ahmad \\ Department of Electronic and Electrical Engineering, University College London, Torrington Place, London WC1E 7JE, United Kingdom \\ W. H. Loh and C. J. Oton \\ Optoelectronics Research Centre, University of Southampton, Highfield, Southampton SO17 1BJ, United Kingdom
}

(Received 20 July 2007; revised manuscript received 31 October 2007; published 15 January 2008)

\begin{abstract}
We discuss the use of rate equations to analyze the sensitization of erbium luminescence by silicon nanoclusters. In applying the general form of second-order coupled rate-equations to the Si nanocluster-erbium system, we find that the photoluminescence dynamics cannot be described using a simple rate equation model. Both rise and fall times exhibit a stretched exponential behavior, which we propose arises from a combination of a strongly distance-dependent nanocluster-erbium interaction, along with the finite size distribution and indirect band gap of the silicon nanoclusters. Furthermore, the low fraction of erbium ions that can be excited nonresonantly is a result of the small number of ions coupled to nanoclusters.
\end{abstract}

DOI: 10.1103/PhysRevB.77.035318 PACS number(s): 78.67. $-\mathrm{n}$, 78.55. $-\mathrm{m}$, 33.80. $-\mathrm{b}$, 32.80. $-\mathrm{t}$

\section{INTRODUCTION}

The sensitization of erbium luminescence by silicon nanoclusters in silicon-rich silica $\left(\mathrm{SiO}_{x}\right)$ is a promising route to silicon-based optical sources and amplifiers operating in the $1.55 \mu \mathrm{m}$ wavelength region. Several groups have demonstrated strong coupling between broad-band absorbing silicon nanoclusters and rare-earth ions - an effect that may lead to the development of efficient broad-band excitable optical sources. ${ }^{1-5}$ Significantly, there has been proof-of-principle demonstration of signal enhancement in an light-emittingdiode-pumped waveguide amplifier based on this material. ${ }^{6}$ Understanding and exploiting the sensitization mechanism relies on the development of an appropriate physical model for the nanocluster-Er interaction. Conventionally, such models are expressed as systems of rate equations describing the populations of the various electronic energy levels in the coupled system. Although there are inevitably a variety of assumptions built into such sets of equations, they are nevertheless very useful tools for modeling the optical properties of the system if they are applied with care.

Rate equations allow various figures of merit to be determined for luminescent systems-radiative and nonradiative lifetimes, interaction coefficients, and cross sections being the most often used. For example, a number of studies have shown that the sensitized erbium luminescence in the $\mathrm{SiO}_{x}$ system may be characterized by an effective excitation cross section $\left(\sigma_{e f f}\right)$ that takes into account absorption of pump photons by $\mathrm{Si}$ nanoclusters, followed by transfer of excitation to nearby erbium ions. ${ }^{4-9}$ In this paper, we discuss the appropriateness of defining an effective cross section and suggest instead that a transfer coefficient is a more robust measure of sensitization.

Moreover, many groups have reported that the fraction of erbium ions that can be excited via the sensitization process is, in fact, very small — of the order of a few percent. ${ }^{10}$ Various reasons have been put forward for this fraction being so small, with competing explanations of excited state absorption causing the nearest neighbor erbium to monopolize the energy transfer from the nanocrystal, ${ }^{11}$ strong distancedependent interactions, ${ }^{12}$ and just a low concentration of donor species amongst those proposed. ${ }^{13}$ A rigorous examination of the interdependence of the populations of the donor and acceptor excited states would help shed further light on the dominance of these various factors and the material parameters that they might be applicable to.

In this paper, we begin by considering a simplified description of the system, with both the silicon nanoclusters and erbium ions treated as quasi-two-level systems. This deliberately simplified case allows us to probe the general behavior of two coupled species-and the limitations of such an approach. We compare the predictions with the results of time-resolved studies of sensitized erbium luminescence, and we show that these results reflect a more complex behavior than a simple rate-equation approach predicts. The rise and fall dynamics of the erbium photoluminescence exhibit a stretched exponential behavior that reflects a distribution of time constants in the system. This distribution can be related to fundamental properties of the silicon nanoclustersspecifically, their indirect band gap and finite size distribution-and to the strong distance dependence of the nanocluster-erbium interaction.

\section{GENERAL FORM OF COUPLED RATE EQUATIONS: TWO-LEVEL SYSTEMS}

We initially look at the simplest system of two two-level systems coupled via an (unspecified) excitation exchange process (Fig. 1). First, we assume that we have two coupled processes: the generation of an initial excited state, $A^{*}$, from a precursor $A$ (donor), and a second excited state, $B^{*}$, generated from a precursor $B$ (acceptor). Assume that an exchange of energy between $A^{*}$ and $B$ produces $B^{*}$. Thus,

$$
\begin{gathered}
A+h \nu \rightarrow A^{*}, \\
A^{*}+B \rightarrow A+B^{*} .
\end{gathered}
$$




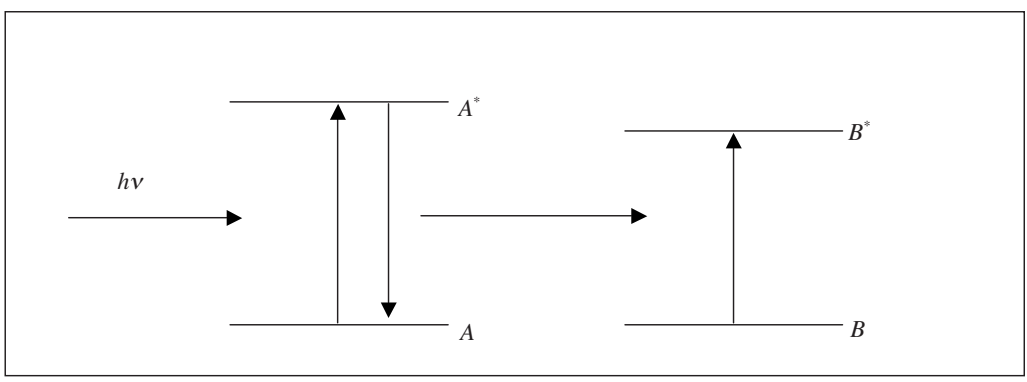

FIG. 1. Schematic representation of two coupled two-level systems under optical excitation.
Further, we assume that backtransfer from $B^{*}$ to $A$ does not occur-in the $\mathrm{Si}$-nc/Er system, this is a reasonable assumption as such processes are not commonly observed under cw excitation conditions due to the energy mismatch between the $A \rightarrow A^{*}$ and $B \rightarrow B^{*}$ transitions, notwithstanding recent reports of Auger deexcitation in samples in the short pulse high flux regime. ${ }^{14}$ Next, we assume that the initial excitation is optical, with a photon flux $\phi$ and an absorption cross section $\sigma$. We further assume that there is a limited reservoir of initial states, $A_{0}$. We can write a rate equation for the generation of $A^{*}$ as

$$
\frac{d A^{*}}{d t}=\sigma \phi\left(A_{0}-A^{*}\right)-\frac{A^{*}}{\tau_{A}} .
$$

Note that $\tau_{A}$ is the effective lifetime of $A^{*}$, which takes into account radiative and nonradiative decay-including transfer to $B$. Solving Eq. (1) for a step excitation pulse gives us

$$
A^{*}=\frac{\sigma \phi \tau_{A} A_{0}}{\sigma \phi \tau_{A}+1}\left\{1-\exp \left[-\left(\sigma \phi+\frac{1}{\tau_{A}}\right) t\right]\right\},
$$

where the reciprocal rise time of the population of $A^{*}$ is a linear function of pump photon flux,

$$
\frac{1}{\tau_{r A}}=\sigma \phi+\frac{1}{\tau_{A}} .
$$

We note that in integrating Eq. (1) in order to arrive at Eqs. (2) and (3), $\tau_{A}$ has been assumed to be a constant, which implies a constant energy transfer to $B$. This can either be considered as an approximation or be rigorously justified by invoking excited state absorption in the transfer process. ${ }^{9}$

Next, we set up a rate equation to describe the excitation of $B$. We shall define a coupling coefficient for the process of transfer of excitation from $A^{*}$ to $B$ as $\gamma$. In contrast to Eq. (1), in which excitation is provided by photon flux, excitation arises in this case via transfer from a population of excited state donors. Thus,

$$
\frac{d B^{*}}{d t}=\gamma A^{*}\left(B_{0}-B^{*}\right)-\frac{B^{*}}{\tau_{B}} .
$$

Once again, $\tau_{B}$ is an effective lifetime that includes radiative and nonradiative contributions. Solving Eq. (4) assuming that the population of $A^{*}$ reaches a steady state well before that of $B^{*}-$ a reasonable approximation as the rise time of $B^{*}$ is typically on the order of a millisecond while that of $A^{*}$ is $10 \mu \mathrm{s}$ or less-gives us

$$
\begin{aligned}
B^{*}= & \frac{\gamma \tau_{B} A_{0} \sigma \phi \tau_{A} B_{0}}{\gamma \tau_{B} A_{0} \sigma \phi \tau_{A}+\sigma \phi \tau_{A}+1} \\
& \times\left\{1-\exp \left[-\left(\gamma \frac{\sigma \phi \tau_{A} A_{0}}{\sigma \phi \tau_{A}+1}+\frac{1}{\tau_{B}}\right) t\right]\right\} .
\end{aligned}
$$

Note now that the reciprocal rise time of the population of excited acceptors is nonlinear in $\phi$,

$$
\frac{1}{\tau_{r B}}=\gamma \frac{\sigma \phi \tau_{A} A_{0}}{\sigma \phi \tau_{A}+1}+\frac{1}{\tau_{B}} .
$$

We consider what it is about the physics of the problem that results in the nonlinearity. Because the excitation of $B$ to $B^{*}$ is a function of the excited state population of donors, the saturation behavior of $A$ is "passed on" to the expressions governing $B^{*}$. The $\sigma \tau_{A}$ term determines the degree of curvature of the function with increasing photon flux, with the steady state value of the first term saturating at $\gamma A_{0}$. If the photon flux becomes high enough to be of the order of $(\sigma \tau)^{-1}$, the curvature becomes significant, and saturation of the reciprocal rise time is evident. Note further that the reciprocal rise time of $B^{*}$ saturates at the same value of photon flux that the population of excited state donors saturates. The degree of curvature of this function is thus not determined by the properties of the acceptor system; rather it is governed solely by the donor species. The dependence is illustrated in Fig. 2, which shows the variation in the reciprocal rise time of $B^{*}$ as a function of photon flux for different values of the cross section of $A$. For illustrative purposes, all parameters other than $\sigma$ have been set to 1 .

A fuller discussion of the validity of this model to the $\mathrm{SiO}_{x}$ : Er system is given in the Appendix. In contrast to our formulation of the rate equations, we note briefly the alternative concept of invoking an effective excitation cross section $\left(\sigma_{e f f}\right)$. $4,7,8,12$ We could choose, as we have done in the past, ${ }^{7}$ to rewrite Eq. (4) as

$$
\frac{d B^{*}}{d t}=\sigma_{e f f} \phi\left(B_{0}-B^{*}\right)-\frac{B^{*}}{\tau_{B}} .
$$

However, in comparing Eqs. (4) and (7), it is clear that $\sigma_{\text {eff }}$ is a function of the excited state population of donors $\left(A^{*}\right)$, which is in turn a function of the photon flux [Eq. (2)]. This variation of $\sigma_{e f f}$ with $\phi$ is unfortunate and tends to mask the physics of the interaction. The strength of the coupling between donors and acceptors depends only on gamma, which is independent of photon flux (second-order effects notwithstanding), while the population of $A^{*}$ depends on the 


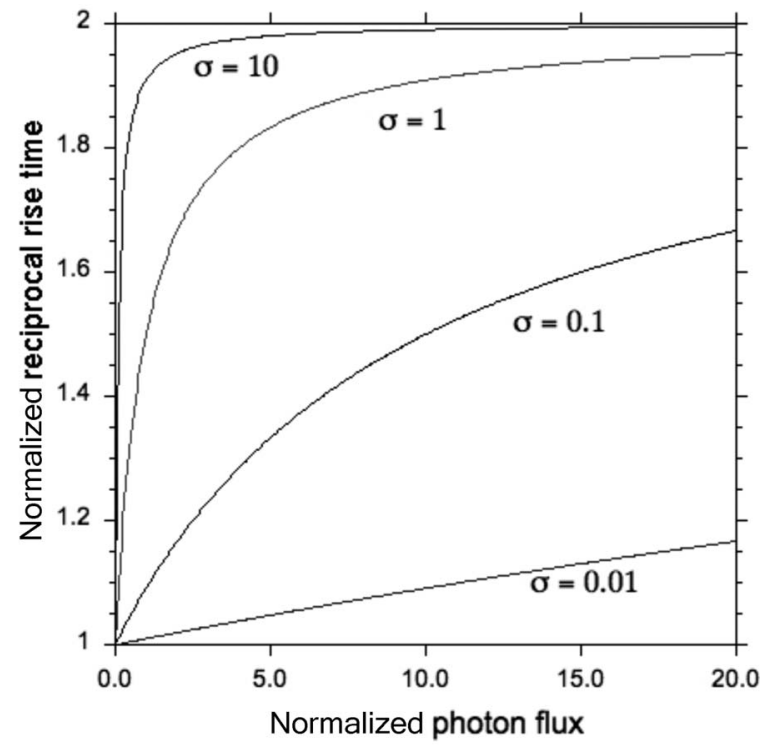

FIG. 2. The dependence of the reciprocal rise time of $B^{*}$ on photon flux for a range of $A$ cross sections [from Eq. (6)]. In this case, the values of $\gamma, A, \tau_{A}$, and $\tau_{B}$ have all been set to 1 .

absorption cross section of donors, donor lifetime, donor concentration, and photon flux. We therefore consider gamma to be a more robust figure of merit than $\sigma_{\text {eff. }}$. Moreover, having units of $\mathrm{cm}^{3} \mathrm{~s}^{-1}$, it is directly comparable to other interaction coefficients such as those describing ion-ion interactions between erbium ions. Defining an effective excitation cross section is misleading insofar as it appears to separate $\phi$ but without actually doing so.

\section{EXPERIMENT}

Samples of erbium-doped silicon-rich silica were deposited by plasma enhanced chemical vapor deposition (PECVD). $\mathrm{SiO}_{x}$ : Er layers with thicknesses of around $1 \mu \mathrm{m}$ were deposited on $\mathrm{Si}(100)$ substrates. Silane $\left(\mathrm{SiH}_{4}\right)$ and nitrous oxide $\left(\mathrm{N}_{2} \mathrm{O}\right)$ were used as precursor gases - the former being diluted to $5 \%$ in argon—and erbium(thd $)_{3}$ vapor with argon as a carrier gas was used to achieve erbium doping during the growth. After deposition, the samples were annealed at $1100{ }^{\circ} \mathrm{C}$ in an argon ambient to precipitate and grow Si nanoclusters. By comparison with material grown under similar conditions, which has been studied using Rutherford backscattering spectroscopy and transmission electron microscopy, we estimate the concentrations of erbium and silicon nanoclusters to be approximately $10^{18}$ and $10^{17} \mathrm{~cm}^{-3}$, respectively. Photoluminescence was excited using an $\mathrm{Ar}^{+}$laser at a wavelength of $\lambda_{\text {exc }}=476 \mathrm{~nm}$ (i.e., nonresonant with Er absorption lines to ensure that Er photoluminescence was stimulated by excitation transfer from sensitizers), analyzed with a Bentham M300 single grating monochromator, and detected with an InGaAs photodiode. To measure time-resolved erbium photoluminescence (PL), the laser was modulated with Pockels cell, and wave forms were recorded with a digital oscilloscope. The time resolution of the system was of the order of $50 \mu \mathrm{s}$. Measure-

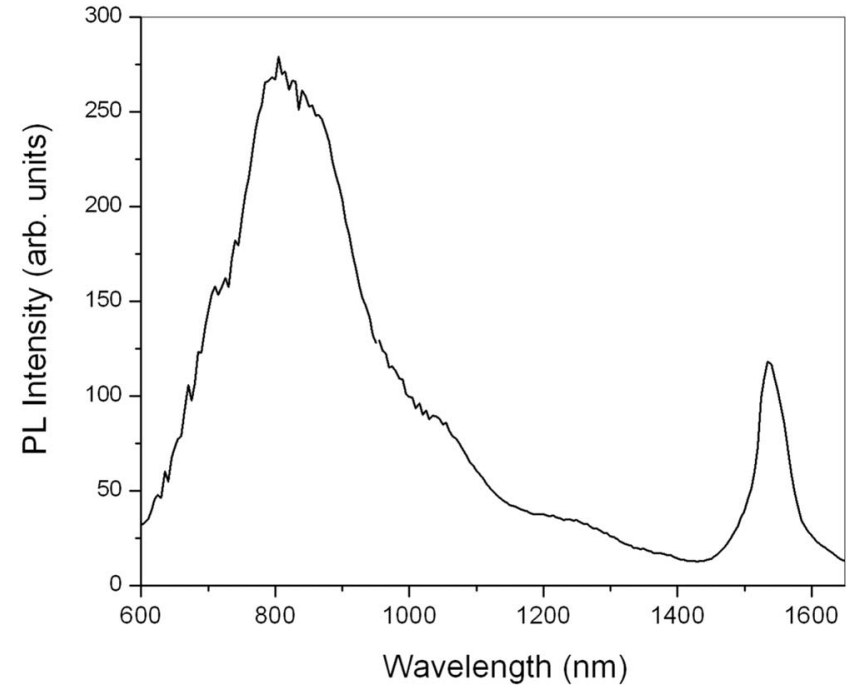

FIG. 3. Photoluminescence of PECVD-deposited sample showing nanocluster emission at $800 \mathrm{~nm}$ and erbium emission around $1535 \mathrm{~nm}$. The spectrum was excited using $476 \mathrm{~nm}$ light and has been corrected for the system response.

ments of Si-nc related PL at $800 \mathrm{~nm}$ were performed with a near-IR-sensitive Hamamatsu photomultiplier, which allows time-resolved measurements with a resolution limited by the bandwidth of the preamplifier, about $5 \mu \mathrm{s}$.

\section{COMPARISON OF THE MODEL WITH EXPERIMENT}

Figure 3 shows a photoluminescence spectrum of the sample, showing luminescence from silicon nanoclusters around $800 \mathrm{~nm}$ and characteristic erbium emission at $1535 \mathrm{~nm}$, as is typically expected.

However, when we try to compare the predictions of the model with time-resolved PL results, it becomes clear that the time dependence of the erbium photoluminescence is not well described by the exponential form of Eq. (5). Figure 4 shows a typical rise curve for $\mathrm{Er}$ photoluminescence at

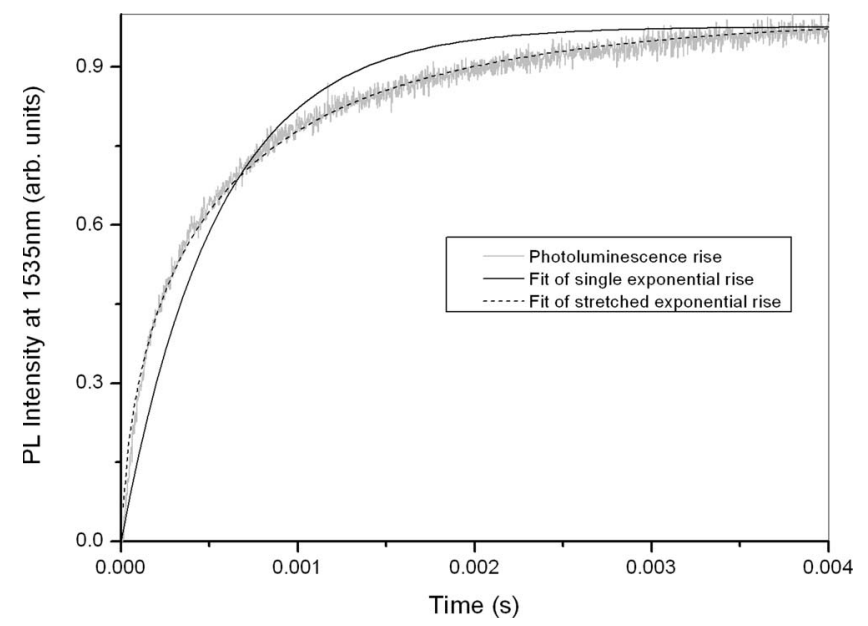

FIG. 4. The rise of Er PL under step excitation, $\lambda_{e x}=476 \mathrm{~nm}$. The solid line is the best fit using a single exponential; the dotted line is a stretched exponential fit. 
$1535 \mathrm{~nm}$ under step excitation using a pump wavelength of $476 \mathrm{~nm}$. The solid line, which represents the best fit using a single exponential rise, is clearly inadequate. In fact, a much better fit is obtained using a stretched exponential function, as defined in Eq. (10) (dotted line),

$$
I=I_{S S}\left\{1-\exp \left[-\left(\frac{t}{\tau_{r B}}\right)^{\beta}\right]\right\}
$$

\section{ANALYSIS AND REFINEMENT OF THE BASIC MODEL}

The stretched exponential decay function has been known since the 19th century as the Kohlrausch law, ${ }^{15}$ which has been found to fit a variety of relaxation processes. Significantly, the decay of photoluminescence from silicon nanocrystals can be successfully described by this function, ${ }^{16}$ and not with a single exponential. Although it is widely used, the physical model for the origin of the stretched exponential function remains somewhat controversial. It is usually explained that this type of relaxation originates from exciton migration between randomly distributed transport and trapping centers, ${ }^{17,18}$ or from ensembles of recombination centers that have different relaxation times. ${ }^{19}$ Both approaches lead to a situation in which radiative recombination at different groups of centers occurs with different relaxation times, which means that the stretched exponential function can be considered a superposition of single exponentials with a certain distribution of relaxation times. Therefore, its expansion into an integral of single exponential functions with a continuous distribution of decay times is possible. ${ }^{20,21}$ Similar reasoning applies to nonexponential rise times. It is important to note that stretched exponential functions of various values of $\beta$ give different continuous distributions of single exponential terms, but an arbitrary superposition of given single exponentials does not necessarily build up into a stretched exponential. Nevertheless, it is possible, at least in principle, to obtain the distribution of time constants present in the system from the stretched exponential function.

Figure 5 presents the reciprocal rise time, obtained from stretched exponential fits to our data, as a function of excitation photon flux. Seemingly, as predicted by our model, this is nonlinear in photon flux. The solid line is a result of fitting using Eq. (6), which gives a value for $\sigma \tau_{A}$ of $4.4 \pm 2.2 \times 10^{-19} \mathrm{~cm}^{2} \mathrm{~s}$. We are also able to obtain the steady state photoluminescence intensity shown in Fig. 6. The solid line is a fit using the preexponential term in Eq. (5). However, the apparently "good" fits are deceptive.

If the data observed are really the consequence of Eqs. (5) and (6), the reciprocal rise time of the erbium luminescence should saturate at the same photon fluxes as the nanocluster luminescence intensity saturates. However, over the range of photon fluxes studied, the characteristic PL from the silicon nanoclusters shows a simple linear increase with photon flux. Moreover, the $\sigma \tau_{A}$ value obtained from fitting to Fig. 5 is unfeasibly large: for a cluster cross section in the region of $10^{-15}-10^{-16} \mathrm{~cm}^{2}$, as is commonly used in the literature, ${ }^{22} \mathrm{we}$ would obtain a lifetime of the order of 0.1-1 ms. Measurements of the nanocluster PL lifetime in our sample showed it

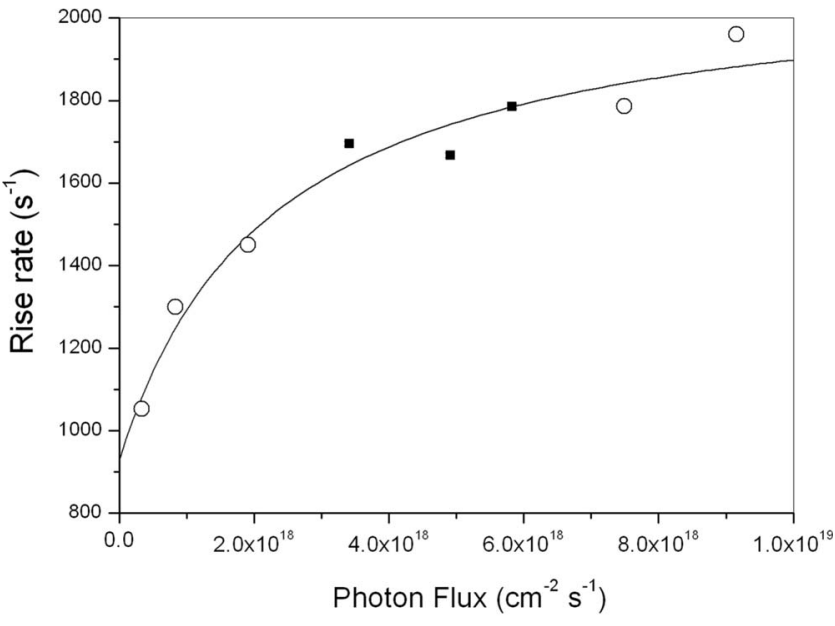

FIG. 5. Rise rate of erbium photoluminescence as a function of excitation photon flux. Values are extracted from a stretched exponential fit to PL wave forms. Circled points are those values of photon flux for which distributions of time constants have been obtained using Eq. (11) and displayed in Fig. 8.

instead to be of the order of our system resolution $(5-10 \mu \mathrm{s})$, which would imply a cross section around $10^{-13} \mathrm{~cm}^{2}$ - such a cross section is simply too high to be plausible. As an aside, it should be noted that there is considerable variation in the luminescence lifetimes of silicon nanoclusters reported in the literature. Nanocluster lifetimes tend to be growth dependent and vary strongly with the degree of crystallinity of the clusters, their size, mean separation, the nature of the matrix surrounding them, and the presence of nonradiative deexcitation pathways. ${ }^{23}$ For example, Cho et al. demonstrated lifetimes between 2 and $60 \mu$ s for $3.4 \mathrm{~nm}$ diameter clusters,${ }^{24}$ and implanted material can show lifetimes between $<5$ and $30 \mu \mathrm{s} .^{25}$ In contrast, longer lifetimes have been reported in the region of $50-100 \mu \mathrm{s},{ }^{26}$ and even as long as $500 \mu$ s for noninteracting nanoclusters. ${ }^{27}$

Clearly, an interpretation of the stretched exponential rise time data requires considerable care. Defining the stretched

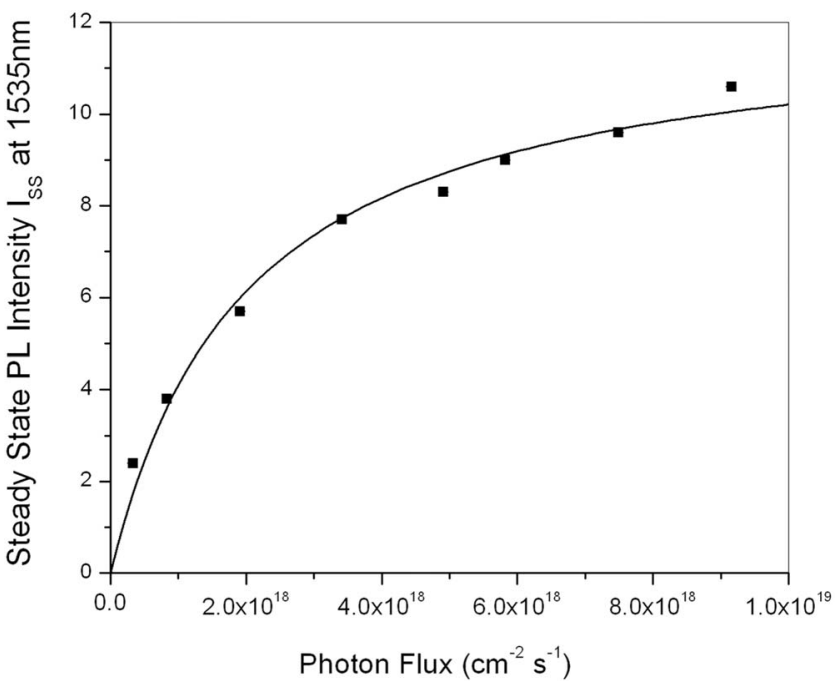

FIG. 6. Steady state intensity of erbium photoluminescence as a function of excitation photon flux. 
exponential as the superposition of a number of separate single exponential terms with a distribution $\rho(\tau), \tau$ being the time constant of any given rise, we find

$I_{s S}\left\{1-\exp \left[-\left(\frac{t}{\tau_{r B}}\right)^{\beta}\right]\right\}=\int_{0}^{\infty} I_{s s}\left\{1-\exp \left[-\left(\frac{t}{\tau}\right)\right]\right\} \rho(\tau) d \tau$

where $\tau_{r B}$ is the observed effective rise time arising from the distribution of $\tau$ values. This is analogous to the more conventionally studied system of a distribution of exponential decays,

$$
A\left\{\exp \left[-\left(\frac{t}{\tau_{\text {effective }}}\right)^{\beta}\right]\right\}=\int_{0}^{\infty} A\left\{\exp \left[-\left(\frac{t}{\tau}\right)\right]\right\} \rho(\tau) d \tau .
$$

The distribution function $\rho(\tau)$ can be obtained by an inverse Laplace transform of the stretched exponential. In the limiting case of $\beta=1, \rho(\tau)$ is a delta function (corresponding to a single exponential), and for a $\beta$ of approximately $0.8, \rho(\tau)$ approximates a Gaussian distribution. For smaller values of $\beta$, the distribution function becomes progressively more skewed toward large values of $\tau$. Beta is thus a measure of the shape of the distribution, although the significance of $\tau_{\text {effective }}$ is harder to quantify. While it is related to the mean of the time constants, it is strictly neither the mean nor the median: the former is not well defined for arbitrary distribution functions, and the latter is not useful for highly skewed distributions with very long tails. A fuller discussion of its significance can be found in Refs. 20 and 21.

A numerical approach to obtaining $\rho(\tau)$, which can be usefully applied to luminescence data, is that adopted by Delerue et $a l .{ }^{28}$ The observed PL decay can be treated as a discrete sum of exponential terms weighted by a distribution of time constants,

$$
I(t)=\sum_{1}^{i} \frac{1}{\tau_{i}} A_{i} \exp \left(-\frac{t}{\tau_{i}}\right)
$$

Determining the distribution of the constants $A_{i}$ over $i$ allows us to obtain an approximation to $\rho(\tau)$. A similar approach can be taken to rise time data. We have performed such an analysis of our time-resolved PL data. We chose 80 values of $\tau$ ranging between 0.01 and $18 \mathrm{~ms}$ and fitted the stretched exponential data obtained from our experimental results using a Levenberg-Marquardt fitting algorithm. We initially confirmed the accuracy of this method by performing fits on analytically generated known distributions of single and multiple exponential data.

Figure 7 shows a plot of the values of beta obtained from fitting a series of erbium PL rise time and decay time data for the sample studied in Figs. 5 and 6 under varying photon fluxes. It is immediately clear that beta for the rise time data varies from approximately 0.75 at low pump fluxes to 0.6 at higher values. Similarly, the decay time data are also characterized by a stretched exponential with a varying beta. However, in this case, the values of beta are higher, and the variation with photon flux is smaller.

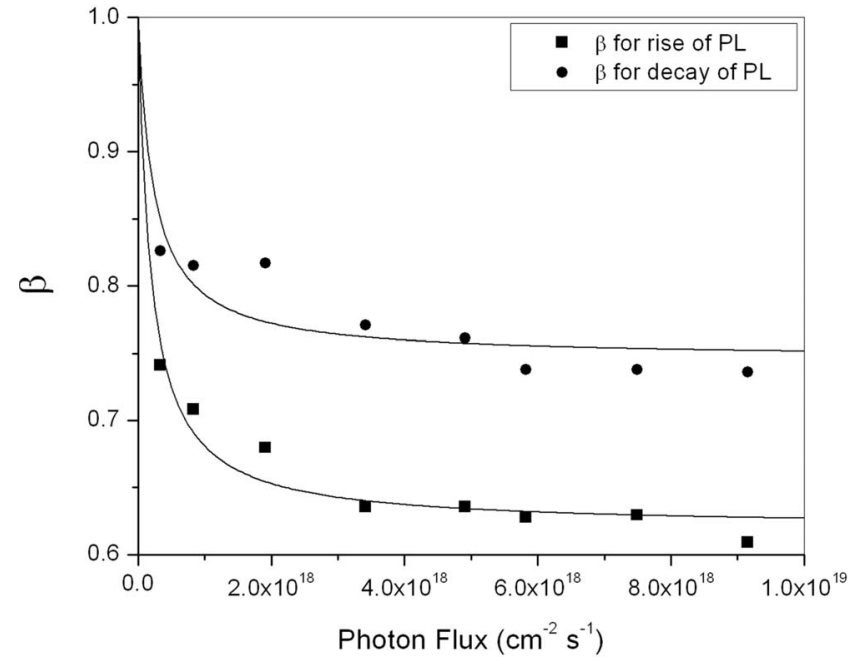

FIG. 7. The variation of the stretched exponential fitting parameter, $\beta$, with $476 \mathrm{~nm}$ pump photon flux for Er PL rise (solid points) and decay (open squares) data from the sample studied in Figs. 6 and 7 . The lines are guides for the eye.

Figure 8 shows the result of fitting the rise time data summarized in Figs. 5 and 7 with a sum of single exponentials. Of particular note is the change in shape of the distribution function with photon flux: at low fluxes, the distribution is almost Gaussian, while at high fluxes, a long tail toward long time constants is apparent, implying that increasing pump photon flux produces slow components in the rise time- this is not predicted by the basic model presented in Eq. (6). Of further note is the difference between the peak values of the distributions and the rise times obtained from the stretched exponential fits shown in Fig. 6. This clearly demonstrates

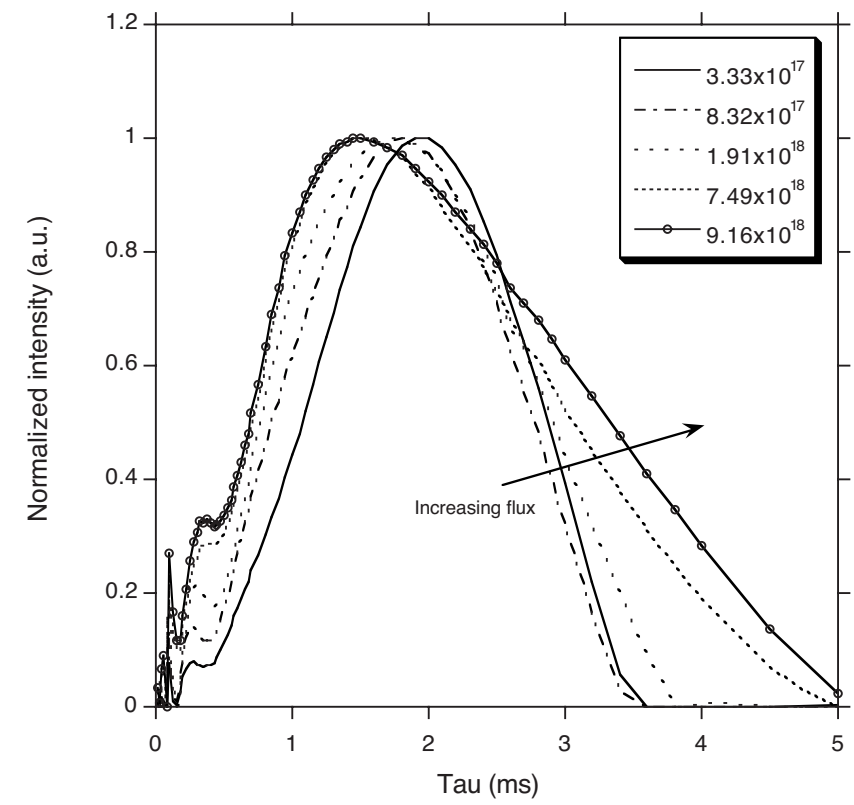

FIG. 8. The distribution of time constants obtained by fitting the rise time stretched exponential data with a set of discrete single exponential functions. Note that all distributions have been normalized to their respective maxima. Each curve represents a different pump photon flux (labeled in the text box). 


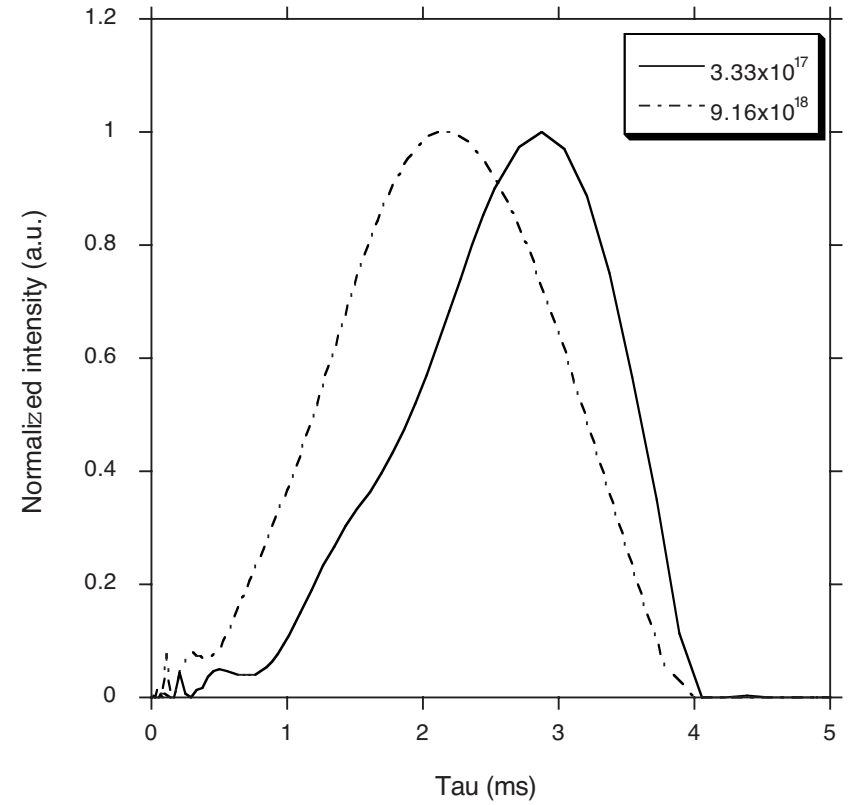

FIG. 9. Distribution functions obtained from fitting Er luminescence decays with a set of discrete single exponentials. The curves have been normalized to their respective maxima. The pump photon fluxes for the two curves are given in the text box.

that the fitting parameter $\tau$ cannot be regarded simply as a mean time constant and illustrates the danger in focusing on changes in $\tau$ without considering corresponding changes in the distribution/weighting of the constituent time constants.

Figure 9 shows corresponding fits to the Er luminescence decay curves - in this case, for clarity, only the highest and lowest pump flux data are shown $\left(9.16 \times 10^{18}\right.$ and 3.33 $\times 10^{17} \mathrm{~cm}^{-2} \mathrm{~s}^{-1}$, respectively). The high flux curve is clearly shifted to short time constants, and the distribution broadened. Note that, here, there is no increase observed in slow components with increasing photon flux.

The differences between the Er PL rise and decay behaviors deserve some explanation. To a good approximation, the decay curves are a result of processes that affect only the acceptors (erbium ions) - i.e., radiative and nonradiative decays, along with some contributions from ion-ion interactions. The rise time curves, on the other hand, probe properties of both donors and acceptors, as they contain information on the saturation behavior of the donors (silicon nanoclusters), the transfer mechanism, and the luminescence properties of the acceptors. We surmise that the distribution of time constants seen in the rise time data is the result of a combination of variations in coupling constants and the inherent distribution of time constants in the luminescence of the silicon nanoclusters. Photoluminescence from silicon nanoclusters typically exhibit stretched exponential decays with beta values around $0.7-0.8$, a behavior variously ascribed to nonzero size distributions, to the migration of excitation, and, more recently by Delerue, to the indirect band gap of the silicon nanoclusters.

Looking at the luminescence decay data, we note that the effect of increasing the photon flux is somewhat different from that seen for the rise time data. The predominant effect is a significant increase in the contribution of short time con- stants. Two possible interpretations present themselves: cooperative upconversion or backtransfer to the silicon nanoclusters. We also point to other recent literature data for luminescence decays in a similar material in which variations in beta were attributed to a scatter in the spacing of erbium ions from silicon nanoclusters. ${ }^{29}$ On the basis of the PL data presented here, we cannot yet determine which effect is most important-suffice it to say that the decay curves are characterized by a distribution of time constants that changes with photon flux in a different way to the rise time data.

Turning to the results presented in Figs. 5 and 6 to explain the saturation of the reciprocal PL rise time at such low values of photon flux, we first consider the concentrations of erbium ions and silicon nanoclusters in our sample. These are approximately $10^{18}$ and $10^{17} \mathrm{~cm}^{-3}$, respectively, from which we can estimate the mean Er-Er and nc-nc separations to be approximately 10 and $21 \mathrm{~nm}$, respectively. Assuming a critical interaction distance of $1 \mathrm{~nm},{ }^{30}$ it is a relatively simple matter to determine from geometrical considerations that only around $1-4 \%$ of erbium ions would be sufficiently close to silicon nanoclusters to experience a sensitization effect. Moreover, each silicon nanocluster coupled to erbium should see just one erbium ion within the interaction volume.

Next, we consider the interpretation of the coupling constant $\gamma$. Our material contains a range of donor-acceptor separations and a range of donor (nanocluster) sizes. We can assume that $\gamma$ exhibits a strong dependence on both. Without specifying either a Coulombic or exchange interaction, we note that the probability of excitation transfer can vary as $r^{-n}$ for the former and $e^{-r}$ for the latter. Even for those ions within the critical interaction range, there will therefore be a strong distance dependence of the interaction. Moreover, a Coulombic interaction between an exciton and an erbium ion will depend on the dipole moment of the exciton, which will in turn depend on the diameter of the silicon nanocluster.

From the physical interpretation of Eq. (13), we can consider that our sample contains a distribution of D-A (donoracceptor) pairs with a range of interaction constants. Describing the interaction constant of the $i$ th class of the D-A pairs as $\gamma_{i}$, there will be a corresponding number of D-A pairs in this class, defined by the concentrations $A_{0 i}$ and $B_{0 i}$, such that

$$
A_{0}=\sum_{i} A_{0 i}
$$

and

$$
B_{0}=\sum_{i} B_{0 i}
$$

It is now clear to see that Eq. (5) should be modified into a sum over all classes $i$,

$$
\begin{aligned}
B^{*}= & \sum_{i}\left(\frac{\gamma_{i} \tau_{B} A_{0 i} B_{0 i} \sigma \phi \tau_{A}}{\gamma_{i} \tau_{B} A_{0 i} \sigma \phi \tau_{A}+\sigma \phi \tau_{A}+1}\right. \\
& \left.\times\left\{1-\exp \left[-\left(\gamma_{i} \frac{\sigma \phi \tau_{A} A_{0 i}}{\sigma \phi \tau_{A}+1}+\frac{1}{\tau_{B}}\right) t\right]\right\}\right) .
\end{aligned}
$$


The contribution of a given coupling coefficient, $\gamma_{i}$, is weighted by the numbers of D-A pairs in that class. As we excite the erbium ions via the silicon nanoclusters, the excited state populations of erbium ions with different coupling constants will saturate at different photon fluxes: Ions with large $\gamma$ will saturate at low fluxes, and those with a small $\gamma$ will saturate at high fluxes. The observed effective rise time of erbium PL at a given peak photon flux will be a combination of the rise times of all of the different classes of erbium ions-some of which will saturate rapidly and some more slowly. As the photon flux increases, the ratio of small$\gamma$ ions to large- $\gamma$ ions will increase, and hence the distribution of time constants within the rising wave form will change. Significantly, the contribution of long time constants will increase with increasing flux, which is consistent with the data presented in Fig. 8. The situation we describe is different from that reported elsewhere in the literature ${ }^{12}$ in that each silicon nanocluster that acts as a sensitizer interacts only with one erbium ion. We do not therefore postulate sequential excitation of erbium ions at successively greater distances by a single nanocluster.

As an indication of the potential usefulness of our formulation, from the form of Eq. (14), we can obtain an estimate concerning the strength of the coupling constant $\gamma_{i}$. We note that the rise time of each component is given by

$$
\frac{1}{\tau_{r i}}=\left(\frac{\gamma_{i} \sigma \phi \tau_{A} A_{0 i}}{\sigma \phi \tau_{A}+1}+\frac{1}{\tau_{B}}\right) .
$$

In order to observe a stretched or multiexponential behavior in the PL response, we would need to have, for at least one class $i$ with $\gamma_{\iota}$ (otherwise, the overall PL behavior would manifest itself as a single exponential with time constant $\tau_{B}$ ),

$$
\frac{\gamma_{i} \sigma \phi \tau_{A} A_{0 i}}{\sigma \phi \tau_{A}+1} \gtrless \frac{1}{\tau_{B}} .
$$

Now, as was pointed out earlier, $\sigma \phi \tau_{A} \ll 1$ for the photon fluxes used in our measurements. In fact, $\sigma \phi \tau_{A} \sim 10^{-3}$ (assuming $\sigma=10^{-16} \mathrm{~cm}^{2}, \phi=5 \times 10^{18} \mathrm{~cm}^{-2} \mathrm{~s}^{-1}$, and $\tau_{A}=2$ $\times 10^{-6} \mathrm{~s}$ ), and Eq. (16) thus reduces to

$$
\gamma_{i} \gtrsim \frac{10^{3}}{A_{0 i} \tau_{B}} .
$$

With $\tau_{B} \sim 10 \mathrm{~ms}$ and $A_{0 i}<10^{17} \mathrm{~cm}^{-3}$, we arrive at the useful conclusion that the coupling strength $\gamma$ between the Si nc and its nearest $\mathrm{Er}$ is comparable to or greater than $10^{-12} \mathrm{~cm}^{3} \mathrm{~s}^{-1}$.

To illustrate the rise time saturation effect directly, we have conducted simulations by modeling a simplified system containing just two classes of erbium ions: class 1 ("near"), which has a $\gamma$ of $10^{-12} \mathrm{~cm}^{3} \mathrm{~s}^{-1}$, and class 2 ("far") with a $\gamma$ of $10^{-13} \mathrm{~cm}^{3} \mathrm{~s}^{-1}$. From geometrical considerations, there will be more erbium ions at larger separations than at smaller distances from silicon nanoclusters, so we have assumed that the population of far ions is five times that of near ions, and we have calculated the effective rise time of the Er PL as obtained from a fit considering the contributions of both populations. The results are shown in Fig. 10 and are compared to our experimental data. We find that the rise rate as a

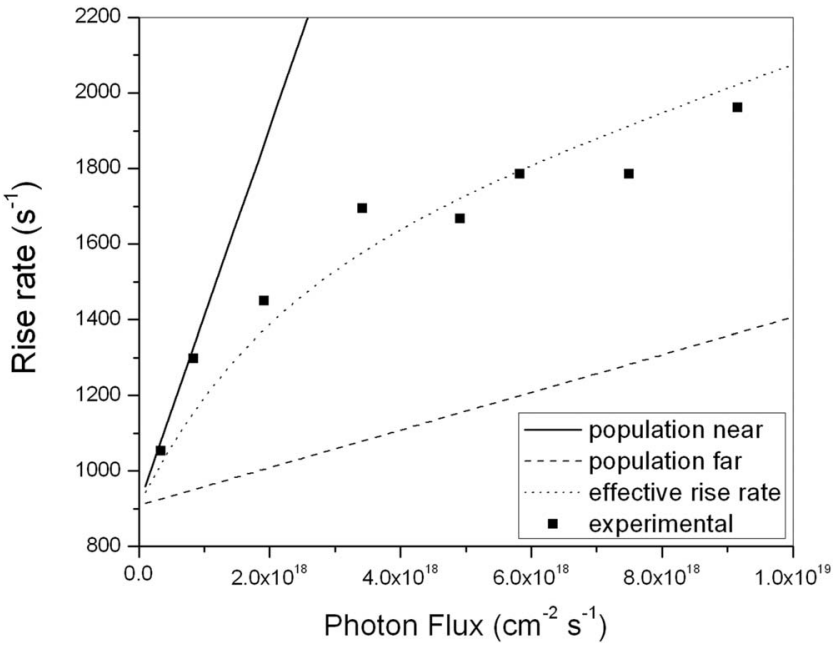

FIG. 10. Simulation of effective rise rate of Er PL from the sum of contributions from ions close to silicon nanoclusters (blue) and far from nanoclusters (green).

function of photon flux is a straight line in both cases for the individual components-as would be expected from Eq. (5) - but the combination of the two together is nonlinear, which is what we would expect from Eq. (14). This arises because at low fluxes the contributions of both classes of ions are equally important, but at high fluxes the far component dominates because the emission from the near ions is saturated. The agreement of the model with experimental results is very good, particularly considering that only two populations of erbium ions have been assumed. In practice, we should assume a weighted quasicontinuous distribution of $\gamma$ values. However, Fig. 10 is a good qualitative illustration of the principle.

We compare the model presented here with the previous model reported by Pacifici et al. in Ref. 8. While that model also applied a rate-equation analysis to the $\mathrm{Si}$ nanocrystal-Er material system, it used the same coupling constant for all of the Si nanocrystals interacting with Er, thereby implicitly assuming that each Si nanocrystal is separated from Er by the same distance. Our current work shows that this is too broad an approximation to account for a range of useful material characteristics. Another, and perhaps more important, difference worth noting is that much of the model in Ref. 8 was devoted to fitting the behavior in material that had a high concentration of $\operatorname{Er}\left(6.5 \times 10^{20} \mathrm{~cm}^{-3}\right)$. At such concentrations, $\mathrm{Er}$ becomes clustered, and $\mathrm{Er}$ cooperative upconversion-an energy loss interaction that serves no useful purpose and needs to be eliminated for viable device applications-became a significant factor and fitting parameter that tended to mask quite a bit of the underlying physics. In contrast, we have deliberately chosen to investigate the behavior of the material with much lower (by 2 orders of magnitude) Er concentration, so that cooperative upconversion is not a complicating factor. This allowed us to focus on the more fundamental material interactions, leading us to the model presented here.

We should point out that the analysis we have performed has been applied only to the erbium photoluminescence. However, a similar examination of the dynamics of the sili- 


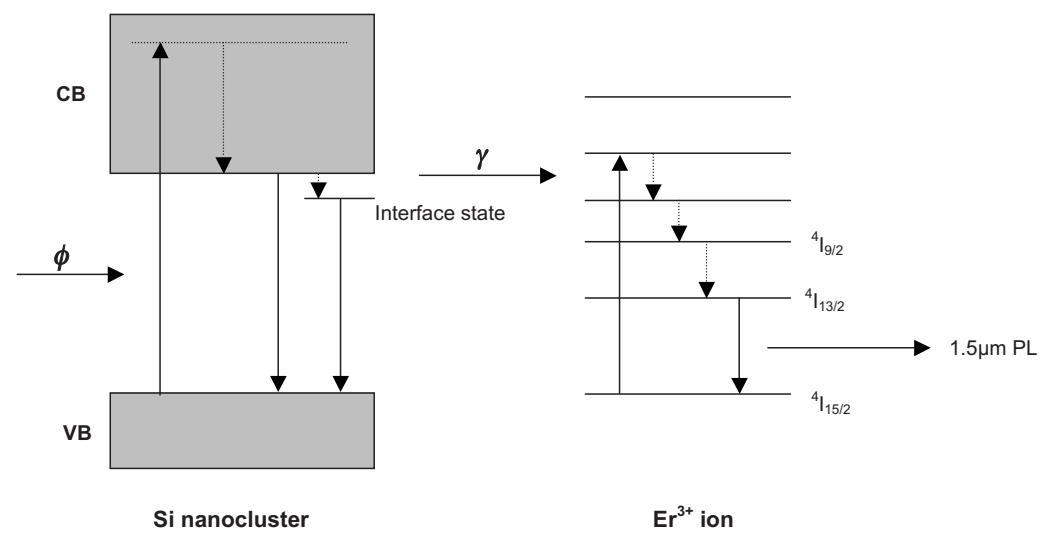

FIG. 11. Schematic diagram of the sensitization of an erbium ion by a silicon nanocluster. A full description is given in the text.

con nanocluster luminescence may also reveal important information on the luminescence mechanism responsible for the $800 \mathrm{~nm}$ emission. A pressing question is whether this PL originates from excitonic recombination within clusters, or if it arises from luminescent centers at the cluster/silica interface. We may expect these two mechanisms to produce very different distributions of time constants in the dynamics of the Si nanocluster PL. It is already known that the luminescence decay from nanoclusters exhibits a stretched exponential form, but to our knowledge there has been no detailed investigation of the rise time dynamics of this PL, which may prove a fruitful area for study. Nevertheless, this falls outside the scope of the work reported here and will be the subject of future investigations.

Finally, we should add a comment on excited state absorption (ESA). In a previous work, we have shown that ESA is a potentially significant effect that enables erbium ions closest to silicon nanoclusters to be repeatedly excited from the metastable state to higher-lying states, ${ }^{11}$ effectively "shielding" the Er ions further away from excitation. The excitation from the metastable to the higher-lying states represents a straight loss mechanism. Moreover, our previous work has suggested that a comparison of the pump photon flux behavior of the nanocluster PL and that from Er can give important information on the presence of ESA; in the absence of ESA, when the Er PL saturates the Si nanocluster PL should increase rather abruptly due to the removal of a nonradiative pathway. However, when ESA is present, such an increase will not be seen, as excited Er ions can still continue to accept energy from the nanoclusters. ${ }^{31}$ We note that our results in this work also show that the nanocluster PL does not increase abruptly when the erbium PL saturates, implying ESA, which will decrease the overall quantum efficiency of the erbium luminescence.

\section{CONCLUSIONS}

We have presented a rate-equation-based model and its generalization to include variations in coupling constants to describe the sensitization of acceptor luminescence by interaction with a donor species. We have shown how this makes important predictions about the temporal behavior of luminescence from the acceptors. In applying this model to the silicon nanocluster/erbium system, we have demonstrated that erbium luminescence kinetics are characterized by a pump flux-dependent distribution of time constants that is likely to arise from a combination of a distribution of coupling coefficients and nonexponential behavior of silicon nanocluster luminescence. The former is likely to arise from the strong distance dependence of the nanocluster-erbium interaction. Furthermore, we have shown that erbium photoluminescence from this material is limited by the low number of erbium ions coupled to silicon nanoclusters, which is a result of the low density of nanoclusters and the short interaction distance. This shortage of sensitization centers results in a low fraction of sensitized erbium ions. We point out that we have not had to invoke effective cross sections or interaction coefficients that vary with photon flux in order to get a good agreement with our experimental observations.

\section{ACKNOWLEDGMENTS}

The authors gratefully acknowledge financial support from the Engineering and Physical Sciences Research Council (EPSRC) and the European Commission as part of the FP6 LANCER project.

\section{APPENDIX: THE $\mathrm{SiO}_{x}$ :Er SYSTEM}

The basic model developed in Sec. II allows us to understand the general features of a coupled system - the different saturation behavior of the donor and acceptor species, and the nonlinear behavior of the acceptor population reciprocal rise time, for example. However, we may examine whether the assumptions that we have made are applicable to the $\mathrm{SiO}_{x}$ : Er system: specifically, whether we are justified in treating the two species as two-level systems.

Figure 11 shows a schematic view of the sensitization mechanism. Absorption of a photon promotes an electron from the valence band of the nanocluster to a high-lying state within the conduction band. This electron then rapidly relaxes to the bottom of the conduction band. If any of the states through which it decays are resonant with energy levels in a nearby erbium ion, the electron can transfer its energy, recombining with a hole in the process. Alternatively, localization of the free carrier at a $\mathrm{Si}=\mathrm{O}$ state at the surface of the nanocluster can occur, from which recombination may also result in excitation of a nearby erbium ion. Such exci- 
tation exchange, by either means, promotes the nearby erbium ion to a high-lying excited state resonant with the energy of the excited electron in the nanocluster, as has been presented by Watanabe et al. ${ }^{32}$ Following this initial excitation step, the excited state Er ion relaxes nonradiatively to the ${ }^{4} I_{13 / 2}$ level from which radiative decay can occur. This nonradiative relaxation is multistep, taking the form of a sequential cascade through the energy levels between the initial level and the ${ }^{4} I_{13 / 2}$ level. Most of these steps are very rapid (nanoseconds), but the rate-limiting step is the transition between the ${ }^{4} I_{11 / 2}$ and ${ }^{4} I_{13 / 2}$ levels, which has a time constant of $2 \mu$ s. This time, which has been measured for the $\mathrm{SiO}_{x}$ : $\mathrm{Er}$ system by several groups, ${ }^{33,34}$ is often referred to as the transfer time, but it is more accurately considered the time for the overall process of excitation, transfer, and sequential relaxation to the ${ }^{4} I_{15 / 2}$ level. The real time taken for the transfer of excitation from nanocluster to erbium ion will be very much shorter.

For the purposes of simplification, we assume that the time taken for a photogenerated carrier to thermalize within the silicon nanocluster to a level from which it interacts with the erbium ion is short enough in comparison to the lifetime of the erbium photoluminescence to be ignored. This thermalization is likely to occur on time scales of the order of picosecond or nanosecond, and we thus treat the silicon nanocluster as a two-level system. Similarly, we further assume that the erbium ion is excited to an (unspecified) highlying excited state, from which the time taken for the excited electron to relax to the ${ }^{4} I_{11 / 2}$ level is much shorter than the lifetime of the ${ }^{4} I_{11 / 2}$ to ${ }^{4} I_{13 / 2}$ transition. As this lifetime is itself very much shorter than that of the ${ }^{4} I_{13 / 2}$ level (by 3 orders of magnitude), we are therefore able to treat the erbium ion as a quasi-two-level system.
*Corresponding author; t.kenyon@ee.ucl.ac.uk

${ }^{1}$ A. J. Kenyon, P. F. Trwoga, M. Federighi, and C. W. Pitt, J. Phys.: Condens. Matter 6, L319 (1994).

${ }^{2}$ M. Fujii, M. Yoshida, Y. Kanzawa, S. Hayashi, and K. Yamamoto, Appl. Phys. Lett. 71, 1198 (1997).

${ }^{3}$ J. H. Shin, M. Kim, S. Seo, and C. Lee, Appl. Phys. Lett. 72, 1092 (1998).

${ }^{4}$ G. Franzò, V. Vinciguerra, and F. Priolo, Appl. Phys. A: Mater. Sci. Process. 69, 3 (1999).

${ }^{5}$ N. Daldosso, D. Navarro-Urrios, M. Melchiorri, L. Pavesi, F. Gourbilleau, M. Carrada, R. Rizk, C. Garcia, P. Pellegrino, B. Garrido, and L. Cognolato, Appl. Phys. Lett. 86, 261103 (2005).

${ }^{6}$ J. Lee, J. H. Shin, and N. Park, J. Lightwave Technol. 23, 19 (2005).

${ }^{7}$ A. J. Kenyon, C. E. Chryssou, C. W. Pitt, T. Shimizu-Iwayama, D. E. Hole, N. Sharma, and C. J. Humphreys, J. Appl. Phys. 91, 367 (2002).

${ }^{8}$ D. Pacifici, G. Franzò, F. Priolo, F. Iacona, and L. Dal Negro, Phys. Rev. B 67, 245301 (2003).

${ }^{9}$ C.-C. Kao, C. Barthou, B. Gallas, S. Fisson, G. Vuye, J. Rivory, A. Al Choueiry, A.-M. Jurdyc, B. Jacquier, and L. Bigot, J. Appl. Phys. 98, 013544 (2005).

${ }^{10}$ M. Wojdak, M. Klik, M. Forcales, O. B. Gusev, T. Gregorkiewicz, D. Pacifici, G. Franzò, F. Priolo, and F. Iacona, Phys. Rev. B 69, 233315 (2004).

${ }^{11}$ C. J. Oton, W. H. Loh, and A. J. Kenyon, Appl. Phys. Lett. 89, 031116 (2006)

${ }^{12}$ B. Garrido, C. Garcia, P. Pellegrino, D. Navarro-Urrios, N. Daldosso, L. Pavesi, F. Gourbilleau, and R. Rizk, Appl. Phys. Lett. 89, 163103 (2006).

${ }^{13}$ P. G. Kik and A. Polman, J. Appl. Phys. 88, 1992 (2000).

${ }^{14}$ I. Izeddin, A. S. Moskalenko, I. N. Yassievich, M. Fujii, and T. Gregorkiewicz, Phys. Rev. Lett. 97, 207401 (2006).

${ }^{15}$ R. Kohlrausch, Ann. Phys. 12, 393 (1847).

${ }^{16}$ J. Linnros, N. Lalic, A. Galeckas, and V. Grivickas, J. Appl. Phys. 86, 6128 (1999).

${ }^{17}$ B. Sturman, E. Podivilov, and M. Gorkunov, Phys. Rev. Lett. 91, 176602 (2003).
${ }^{18}$ L. Pavesi, J. Appl. Phys. 80, 216 (1996).

${ }^{19}$ A. F. van Driel, I. S. Nikolaev, P. Vergeer, P. Lodahl, D. Vanmaekelbergh, and W. L. Vos, Phys. Rev. B 75, 035329 (2007).

${ }^{20}$ C. P. Lindsey and G. D. Patterson, J. Chem. Phys. 73, 3348 (1980).

${ }^{21}$ D. C. Johnston, Phys. Rev. B 74, 184430 (2006).

${ }^{22}$ D. Kovalev, J. Diener, H. Heckler, G. Polisski, N. Künzner, and F. Koch, Phys. Rev. B 61, 4485 (2000).

${ }^{23}$ G. Allan, C. Delerue, and M. Lannoo, Phys. Rev. Lett. 78, 3161 (1997).

${ }^{24}$ E.-C. Cho, M. A. Green, G. Conibeer, D. Song, Y.-H. Cho, G. Scardera, S. Huang, S. Park, X. J. Hao, Y. Huang, and L. V. Dao, Adv. OptoElectron. 2007, 69578.

${ }^{25}$ D. I. Tetelbaum, O. N. Gorshkov, A. V. Ershov, A. P. Kasatkin, V. A. Kamin, A. N. Mikhaylov, A. I. Belov, D. M. Gaponova, L. Pavesi, L. Ferraioli, T. G. Finstad, and S. Foss, Thin Solid Films 515, 333 (2006).

${ }^{26}$ M. Dovrat, Y. Goshen, J. Jedrzejewski, I. Balberg, and A. Sa'ar, Phys. Rev. B 69, 155311 (2004).

${ }^{27}$ O. Guillos, N. Herlin-Boime, C. Reynaud, G. Ledoux, and F. Huisken, J. Appl. Phys. 95, 3677 (2004).

${ }^{28}$ C. Delerue, G. Allan, C. Reynaud, O. Guillois, G. Ledoux, and F. Huisken, Phys. Rev. B 73, 235318 (2006).

${ }^{29}$ D. M. Zhigunov, O. A. Shalygina, S. A. Teturokov, V. Yu. Timoshenko, P. K. Kashkarov, and M. Zacharias, Semiconductors 40, 1193 (2006).

${ }^{30}$ J. H. Jhe, J. H. Shin, K. J. Kim, and D. W. Moon, Appl. Phys. Lett. 82, 4489 (2003).

${ }^{31}$ W. H. Loh and A. J. Kenyon, IEEE Photonics Technol. Lett. 18, 289 (2006).

${ }^{32}$ K. Watanabe, M. Fujii, and S. Hayashi, J. Appl. Phys. 90, 4761 (2001).

${ }^{33}$ M. Fujii, K. Imakita, K. Watanabe, and S. Hayashi, J. Appl. Phys. 95, 272 (2004).

${ }^{34}$ M. Falconeri, E. Borsella, L. De Dominicis, F. Enrichi, G. Franzò, F. Priolo, F. Iacona, F. Gourbilleau, and R. Rizk, Appl. Phys. Lett. 87, 061109 (2005). 